\title{
Spectral correlation and noise reduction in laser filaments
}

Pierre Béjot $^{1,2}$, Jérôme Kasparian ${ }^{2, *}$, Estelle Salmon ${ }^{2}$, Roland Ackermann ${ }^{2}$, Jean-Pierre Wolf ${ }^{1}$

${ }^{1}$ GAP, Université de Genève, 20 rue de l'École de médecine, CH 1211 Genève, Switzerland

${ }^{2}$ LASIM, UMR CNRS 5579, Université Lyon 1, 43 bd du 11 Novembre 1918, F69622 Villeurbanne Cedex, France

\begin{abstract}
Intensity correlations and noise reduction are observed and characterized in the broadband supercontinuum generated by spatio-temporal solitons propagating in air, i.e. in filamentation of ultrashort laser pulses. Large correlations and reduction of the laser noise are observed already at the first steps of the filamentation process, while further propagation results in cascaded $\chi^{(3)}$ broadening processes and yield complex correlation maps. The spectral range yielding an optimal laser noise reduction of $3.6 \mathrm{~dB}$ is found to cover $10 \mathrm{~nm}$ around the fundamental wavelength.
\end{abstract}

\section{PACS}

42.65.Jx Beam trapping, self-focusing, and thermal blooming; 42.65.Tg Optical solitons; nonlinear guided waves; 42.65.Ky Harmonic generation, frequency conversion; 42.50.Lc Quantum fluctuations, quantum noise, and quantum jumps

\section{Introduction}

Recent studies on optical fibers showed that spectral correlations and squeezing occur in temporal solitons $[1,2,3,4,5,6,7,8]$. The origin of correlations in the intensity fluctuations within the white light continuum is intrinsic to spectral broadening by $\chi^{(3)}$ Kerr effect $[9,10]$. Such $\chi^{(3)}$ broadening includes both self-phase modulation (SPM), cross-phase modulation (CPM) and four-wave mixing (FWM). SPM and CPM correspond to the deformation of the temporal envelope of the pulse, while FWM describes the interaction of two incident waves at 
wavelengths $\lambda_{0}$ and $\lambda_{0}{ }_{0}$, which are converted into a pair of photons at the conjugated wavelengths $\lambda_{1}$ and $\lambda_{2}$, for which the energy conservation imposes $1 / \lambda_{0}+1 / \lambda^{\prime}{ }_{0}=1 / \lambda_{1}+1 / \lambda_{2}$. Both SPM and FWM result in typical correlation maps within the spectrum at the exit of the fiber. The wavelengths within the side of the continuum appear anticorrelated with the incident one, while pairs of conjugated wavelengths are strongly correlated [1]. Such correlation maps are very well reproduced by numerical simulations in optical fibers [11]. These simulations also show that photons originating from $\chi^{(3)}$ broadening can in turn undergo subsequent cascaded $\chi^{(3)}$-induced events. The resulting depletion of the corresponding wavelengths results in a more complex correlation map within the continuum [12]. This process occurs at further propagation distances and higher pulse energy, i.e. for higher order solitons. For sufficiently short pulses, phase correlations have been observed within the continuum, i.e. the continuum has a high coherence $[13,14]$, although such phase correlations are beyond the scope of the present work.

Although remaining in the classical domain, intensity correlations within the white-light continuum, as well as laser noise compression, have also recently been observed in the case of spatio-temporal solitons, namely self-guided filaments generated in the air by high-power, ultrashort laser pulses [15]. Filaments [16] arise in the non-linear propagation of ultrashort, high-power laser pulses in transparent media. They result from a dynamic balance between Kerr-lens focusing and defocusing by self-induced plasma generation. In the atmosphere, filaments have been observed over several hundreds of meters, up to a few kilometers away from the laser source [17]. They can be generated and propagated even in perturbed conditions such as clouds [18] or turbulence [19]. These properties open the way to atmospheric applications [20], such as Lidar remote sensing, Laser-induced breakdown spectroscopy (LIBS) [21], lightning control [22] and free space communications. For example, high sensitivity multi-pollutant detection with a "white-light lidar" [20] would greatly benefit of a reduced shot-to-shot noise on the supercontinuum intensity due to the recently observed noise reduction [15].

As described above, the generation of the supercontinuum originates from cascaded $\chi^{(3)}$ events. At the beginning of the process, the fundamental radiation $\omega_{0}$ generates photon pairs at $\omega_{1}$ and $\omega_{2}$, such that $2 \omega_{0}=\omega_{1}+\omega_{2}$. However, once the continuum intensity is sufficient, the generated wavelengths in turn can undergo four-wave mixing. Therefore, a given wavelength 
can be generated through a large number of pathways and correlations within the spectrum may be reduced or even lost after some propagation distance.

In this paper, we extend the previous experimental results [15] by investigating the build-up and the evolution of correlations observed within the spectrum of the white-light continuum, with propagation and for increasing beam power. These results are compared with simple simulations based on SPM. Moreover, the influence of the integration window within the spectrum of the supercontinuum is investigated, providing an optimal filtering scheme for laser noise reduction in laser filaments.

\section{Experimental setup}

The experimental setup is depicted in Figure 1. A CPA (Chirped Pulse Amplification) Ti:Sapphire laser system delivered $200 \mathrm{fs}$ pulses at $22.5 \mathrm{~Hz}$ repetition rate, centered at $815 \mathrm{~nm}$, with $\sim 10 \mathrm{~mm}$ beam diameter (at $1 / e^{2}$ level). The pulse energy could be varied from 1 to $6 \mathrm{~mJ}$. The beam was focused by a spherical mirror with $5 \mathrm{~m}$ focal length, yielding a nonlinear focus (filament onset) $\sim 3 \mathrm{~m}$ downstream of the spherical mirror. The spherical mirror was defined as the origin of the propagation axis $(z=0)$. The filament length was $\sim 4 \mathrm{~m}$. At given distances $z$ comprised between 2 and $12 \mathrm{~m}$, the beam was scattered on a spectrally neutral target, and the light was collected with a fiber into a spectrometer providing $0.3 \mathrm{~nm}$ resolution between 725 and $900 \mathrm{~nm}$. In each experimental condition, 5000 spectra were recorded, normalized to 1 and used to compute the cross-correlation map of the intensity fluctuations across the spectrum. The correlation between two wavelengths $\lambda_{1}$ and $\lambda_{2}$, was calculated as:

$$
C\left(\lambda_{1}, \lambda_{2}\right)=\frac{V\left(n_{1}+n_{2}\right)-\left(V\left(n_{1}\right)+V\left(n_{2}\right)\right)}{2 \cdot \sqrt{V\left(n_{1}\right) \cdot V\left(n_{2}\right)}}
$$

where $V(x)$ is the variance of variable $x$ and $n_{i}$ the photon number (or, equivalently, the intensity), at the wavelength $\lambda_{i}$. 


\section{Results and discussion}

\section{Correlations of the intensity fluctuations}

Figure 2 displays the map of cross-correlations of the intensity fluctuations before the onset of the filaments $((\mathrm{a}), z=2 \mathrm{~m})$, shortly after it $((\mathrm{b}), z=3.5 \mathrm{~m})$, and at the filament end ((c), $z=8 \mathrm{~m}$ ). Before filamentation, correlations are limited to the region corresponding to $\lambda_{1}=\lambda_{2}$. The regions of negative correlation are due to a slight jitter of the fundamental wavelength, as suggested by numerical simulations (see below). Once filamentation occurs ( $z \geq 3 \mathrm{~m}$, i.e. only $0.5 \mathrm{~m}$ propagation within the filament, see Figure $2 \mathrm{~b}$ ), SPM is initiated, and positive correlations are observed in regions corresponding to nearly conjugated wavelengths $\left(2 \omega_{0}=\right.$ $\left.\omega_{1}+\omega_{2}\right)$. In contrast, negative correlations form a dark cross centered on the fundamental wavelength. In other words, the generation of the white-light photons requires depletion of the number of photons about the fundamental wavelength. This behavior is the signature for a simultaneous generation of both the Stokes and the anti-Stokes components of the continuum originating from the depletion of the fundamental wavelength of the incident laser [15]. Further propagation of the filament (Figure 2c) results in a more complicated structure of the correlation map, with stripes of positive and negative correlations appearing around the fundamental wavelength. These stripes are due to the typical oscillatory structure of the supercontinuum generated by $\chi^{(3)}$ broadening, which results from the beating of the waves generated at each wavelength in two slices of the pulse which have the same time derivative of the intensity [23]. After the filament end, the correlation map does not evolve anymore since the laser intensity is too low to allow further $\chi^{(3)}$ broadening.

The same evolution is observed when the laser power is progressively increased while the measurement is performed at a fixed position downstream of the filament $(z=12 \mathrm{~m}$, Figure 3). At low input beam power $(1.3 \mathrm{~mJ} /$ pulse, i.e. $6.5 \mathrm{GW}$, only twice the critical power for filamentation in air [24]), the pattern typical of a single-step SPM process (Figure 3a) is observed. Increasing the laser power allows cascaded $\chi^{(3)}$ broadening events resulting in much more complex patterns (Figure 3b, $9 \mathrm{GW}$ ), and even the disappearance of the correlation between conjugated wavelengths (Figure $3 \mathrm{c}, 16 \mathrm{GW}$ ). These complex patterns correspond to highly structured spectra of the continuum, as displayed under the correlation maps of Figure 3. 
These results show that intensity correlations within the spectrum of the white-light supercontinuum are generated in the course of self-guided filamentation. The absorption and (incoherent) scattering of light by the plasma which is generated within the filaments does not prevent correlations within the spectrum of the continuum. This is further confirmed by comparing the observed correlations maps (see e.g. Figure $2 b$ ) with numerical simulations for temporal solitons (i.e. in fibers) performed by Schmidt et al. [11]: Although they do not take ionization nor other higher order processes into account, these simulations yield the same characteristic pattern in which each wavelength is correlated with its conjugate but anticorrelated with the fundamental wavelength.

The agreement of these calculations with our experimental results is fairly good, although the spatial resolution of our experiment does not allow observing the longitudinal fluctuations of the correlation map. Moreover, according to the simulations, the square regions with positive correlation in Figure 2, as well as the positive stripes within the negative correlation cross, stem from higher-order solitons. Now, we observe experimentally such patterns for high powers and/or long propagation distances, i.e. when cumulative broadening occurs.

To further assess the critical role of $\chi^{(3)}$ broadening in the generation of correlations, we performed a simple simulation of the correlation map. Here, the propagation of the selfguided filament is not described in detail. Instead, only the effect of laser noise on SPM generation is considered. The initial electrical field is defined by its carrier frequency and envelope:

$$
E(z, t)=E_{0} \cdot \cos \left(\omega_{0} \cdot t-\Delta \phi(z, t)\right) \cdot \exp \left(-t^{2} / 2 \cdot \tau^{2}\right)
$$

where $t=T-z / c$ is the reduced time, $T$ is the absolute time, $z$ is the position along the propagation axis, $c$ is the velocity of light, $\omega_{0}$ the frequency of the carrier wave, and $\tau$ the pulse duration. The envelope $E(t)=E_{0} \exp \left(-t^{2} / 2 \cdot \tau^{2}\right)$ is used to compute the dephasing $\Delta \phi(z, t)$ due to Kerr effect after a propagation distance $z$ for each temporal slice of the pulse:

$$
\Delta \phi(z, t)=n_{2} \cdot I \cdot z=n_{2} \cdot E^{2}(t) \cdot z
$$


where $n_{2}$ is the non-linear refractive index $\left(n_{2}=410^{-19} \mathrm{~cm}^{2} / \mathrm{W}\right.$ in the air at $800 \mathrm{~nm} \mathrm{[25]).} \mathrm{A}$ Fourier transform of the deformed carrier yields the resulting spectrum. To simplify the calculation, we consider that the intensity within the filament is strictly clamped for a given shot [26], so that the SPM-generated spectrum can be calculated within one iteration.

We repeated the calculation for initial intensities and wavelengths randomly fluctuating around the nominal parameters of the experiments (200 fs pulses at $815 \mathrm{~nm}$, with a mean intensity of $3.3 \times 10^{16} \mathrm{~W} / \mathrm{m}^{2}$ ). The intensity was normally distributed with a standard deviation of $4.3 \times 10^{15} \mathrm{~W} / \mathrm{m}^{2}$, as estimated from the experimental data. Moreover, a slight jitter of $0.3 \mathrm{~nm}$ on the central wavelength has been added. This fluctuation corresponds to a jitter of the central wavelength between two pixels of the spectrometer. The resulting set of 500 simulated spectra yielded a correlation map, calculated with the same procedure as for experimental data. Figure 4 displays the resulting correlation maps for several propagation distances, similar to those of the experimental results displayed in Figure 2, namely before filamentation, $0.5 \mathrm{~m}$ after the beginning of filamentation and at the filament end, respectively.

The calculated results are very similar to the experimental ones, showing that the dominant process is actually SPM and that the ionization does not jeopardize the correlations in spite of its higher order nonlinearity and lack of coherence (as ionization is not taken into account). Note that the large regions of positive correlation away from the fundamental wavelength stem from the fact that, away from the oscillatory spectrum around the fundamental, both wings of the continuum are generated simultaneously, all the more efficiently that the incident intensity is higher.

\section{Laser noise reduction}

The occurrence of correlations within the spectrum allows us to expect noise reduction for adequately selected spectral regions. We have recently reported a laser noise reduction of $1.2 \mathrm{~dB}$ in filaments, even though the filter (flat gate open from 785 to $820 \mathrm{~nm}$ ) was not optimized [15]. The noise reduction of the spectrally filtered filament supercontinuum is defined as $-10 \cdot \log \left(\frac{V\left(I_{\text {filament }}\right)}{V\left(I_{\text {ref }}\right)}\right)$, where $I_{\text {filament }}$ and $I_{\text {ref }}$ are the intensities associated with the filament and the unfocused laser beam taken as reference, respectively, integrated over the considered spectral interval. In order to estimate the highest level of compression that would 
be achievable using filaments, we sought for an optimal filter, restricting ourselves to square bandpass filters within the $750-880 \mathrm{~nm}$ spectral range, and considering experimental data with $6.5 \mathrm{GW}$ peak power, corresponding to Figure 2. The spectral range yielding maximal noise reduction is $809-819 \mathrm{~nm}$, i.e. centered on the incident laser wavelength $(815 \mathrm{~nm})$. This range is independent of the propagation distance within the filament. The noise reduction over this optimal spectral range increases from $0.69 \mathrm{~dB}$ shortly after the filament onset, to $3.6 \mathrm{~dB}$ at the filament end. This value is three times more than reported in [15]. Moreover, it is comparable with the squeezing observed in fibers after correction for the detection losses (e.g., $4.1 \mathrm{~dB}$ in microstructured fibers [27] and 3.2 or $3.7 \mathrm{~dB}$ for classical fibers $[28,29]$ ), although in our experiment the conditions are far from sub-shot noise. Therefore, soliton propagation in laser-generated self-guided filaments can be expected to be a good candidate for laser-noise reduction.

\section{Conclusion}

As a summary, we have characterized the correlations of the intensity fluctuations and the noise reduction observed in the broadband supercontinuum generated by spatio-temporal solitons propagating in air (filamentation). Large correlations and reduction of the noise is observed already at the first steps of the filamentation process, before multi-step $\chi^{(3)}$ broadening. Multi-step $\chi^{(3)}$ broadening results in more complex correlation patterns without reducing the maximum correlation values. An optimal noise squeezing of $3.6 \mathrm{~dB}$ is obtained for a $10 \mathrm{~nm}$ wide spectral range centered on the fundamental wavelength.

\section{Acknowledgements}

We gratefully acknowledge N. Gisin, H. Zbinden (Université de Genève), C. Fabre (ENS, Paris), G. Leuchs (Universitaet Erlangen) and J.Dudley (Université de Franche-Comte) for very fruitful discussions.

\section{References}

1 S. Spälter, N. Korolkova, F. König, A. Sizmann, G. Leuchs, Observation of Multimode Quantum Correlations in Fiber Optical Solitons. Phys.Rev.Lett. 81, 786, (1998) 
2 T. Opatrny, N. Korolkova, and G. Leuchs, Mode Structure and Photon Number Correlations in Squeezed Quantum Pulses. Phys.Rev.A 66, 053813 (2002)

3 S.J. Carter, P.D. Drummond, M.D. Reid, R.M. Shelby, Squeezing of quantum solitons, Phys.Rev.Lett, 58, 1841 (1987)

4 M. Rosenbluh and R.M. Shelby, Squeezed optical solitons, Phys. Rev. Lett. 66, 153$156(1991)$

$5 \quad$ K. Bergman and H.A. Haus, Squeezing in fibers with optical pulses, Opt.Lett. 16, 663$665(1991)$

6 K. Bergman, C.R. Doerr, H.A. Haus and M.Shirasaki, Sub-shot-noise measurement with fiber squezzed optical pulses, Opt.Lett. 18, 643-645 (1993)

7 K. Bergman, H.A. Haus, E.P. Ippen and M.Shirasaki, Squeezing in a fiber interferometer with a gigahertz pump, Opt.Lett. 19, 290-292 (1994)

8 N. Nishizawa, S. Kume, M. Mori, T.Goto and A.Miyauchi, Squeezed light generation with 1.064 um Nd:YAG laser and 0.85 um single-mode fiber, Jpn.J.Appl.Phys, Part 1 33, 138-143 (1994)

9 L. Boivin, F. X. Kärtner, H. A. Haus, Analytical Solution to the Quantum Field Theory of Self-Phase Modulation with a Finite Response Time, Phys.Rev.Lett., 73, 240, (1994)

10 A. Mecozzi and P. Kumar, Linearized quantum-fluctuation theory of spectrallyfiltered optical solitons, Opt. Lett. 22, 1232-1234 (1997) 
11 E. Schmidt, L. Knöll, D.-G. Welsch, Cumulant expansion for studying damped quantum solitons, Physical Review A 59, 2442 (1999)

12 E.Schmidt, L.Knöll, D.-G.Welsch, M.Zielonka, F.König and A.Sizmann, Physical Review Letters 85, 3801 (2000)

13 M. Bellini, T. W. Hänsch, Phase-locked white-light continuum pulses : towards a universal optical frequency-comb synthesizer, Optics Letters 25, 1049 (2000)

14 J. W. Nicholson, M. F. Yan, Cross-coherence measurements of supercontinua generated in highly non-linear, dispersion shifted fiber at $1550 \mathrm{~nm}$, Optics Express 12, 679 (2004)

15 Pierre Béjot, Jérôme Kasparian, Estelle Salmon, Roland Ackermann, Nicolas Gisin, Jean-Pierre Wolf, Laser Noise Reduction in Air, Applied Physics Letters 88, 251112 (2006)

16 A. Braun, G. Korn, X. Liu, D. Du, J. Squier, and G. Mourou, Self-channeling of highpeak-power femtosecond laser pulses in air. Optics Letters 20, 73 (1995).

17 M. Rodriguez, R. Bourayou, G. Méjean, J. Kasparian, J. Yu, E. Salmon, A. Scholz, B. Stecklum, J. Eislöffel, U. Laux, A.P. Hatzes, R. Sauerbrey, L. Wöste, and J.-P. Wolf, Kilometer-range non-linear propagation of femtosecond laser pulses. Physical Review E 69, 036607 (2004).

18 G. Méjean, J. Kasparian, J. Yu, E. Salmon, S. Frey, J.-P. Wolf, S. Skupin, A. Vinçotte, R. Nuter, S. Champeaux, and L. Bergé, Multifilamentation transmission through fog. Physical Review E 72, 026611 (2005). 
19 R. Ackermann, G. Méjean, J. Kasparian, J. Yu, E. Salmon, and J.-P. Wolf, Laser filaments generated and transmitted in highly turbulent air. Optics Letters. 31, 86 (2006)

20 J. Kasparian, M. Rodriguez, G. Méjean, J. Yu, E. Salmon, H. Wille, R. Bourayou, S. Frey, Y.-B. André, A. Mysyrowicz, R. Sauerbrey, J.-P. Wolf, and L. Wöste, WhiteLight Filaments for Atmospheric Analysis. Science 301, 61-64 (2003).

21 K. Stelmaszczyk, P. Rohwetter, G. Méjean, J. Yu, E. Salmon, J. Kasparian, R. Ackermann, J.-P. Wolf, and L. Wöste, Applied Physics Letters 85, 3977 (2004)

22 R. Ackermann, K. Stelmaszczyk, P. Rohwetter, G. Méjean, E. Salmon, J. Yu, J. Kasparian, G. Méchain, V. Bergmann, S. Schaper, B. Weise, T. Kumm, K. Rethmeier, W. Kalkner, J. P. Wolf, and L. Wöste, Applied Physics Letters 85, 5781-5783 (2004).

23 G. P. Agrawal, Nonlinear Fiber Optics, Third Edition, Academic Press, p. 102 (1995)

24 Y. R. Shen, The principles of nonlinear optics (John Wiley \& Sons, New-York, 1984)

25 X. M. Zhao, P. Rambo, J.-C. Diels, Effect of oxygen on the laser triggering of lightning, QELS'95 16, 178 (1995)

26 A. Becker, N. Aközbek, K. Vijayalakshmi, E. Oral, C.M. Bowden, and S.L. Chin, Intensity clamping and re-focusing of intense femtosecond laser pulses in nitrogen molecular gas. Applied Physics B. 73, 287-290 (2001)

27 M. Fiorentino, J. E. Sharping, P. Kumar, A. Porzio, R. S. Windeler, Optics Letters 27, $649(2002)$ 
28 S. Spälter, M.Burk, U.Strößner, A.Sizmann, G.Leuchs, Propagation of quantum properties of sub-picosecond solitons in a fiber, Optics Express, Vol.2, No. 3, 77 (1998)

29 S. R. Friberg, S. Machida, M. J. Werner, A. Levanon, T. Mukai, Physical Review Letters 77, 3775 (1996) 


\section{Figures}

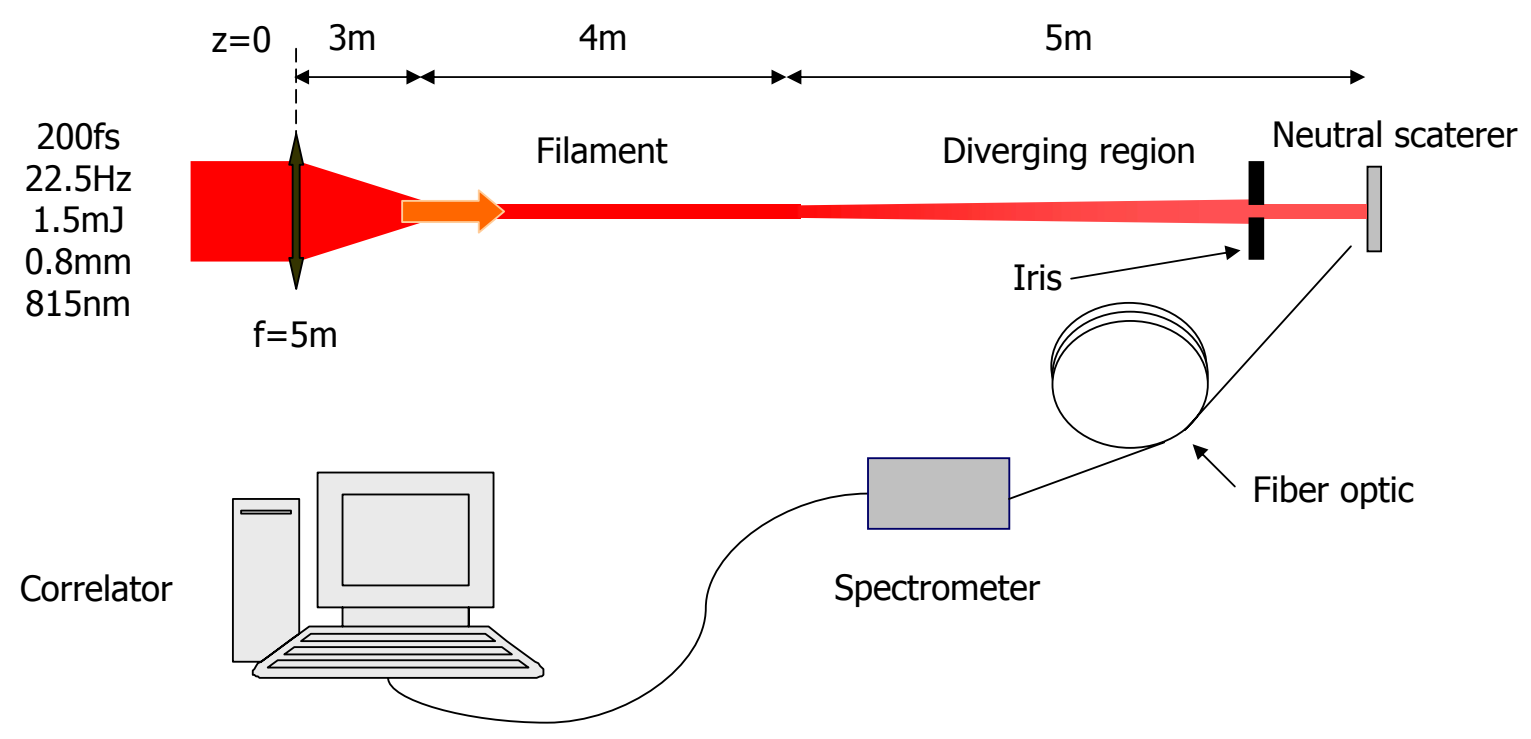

Figure 1. Experimental setup
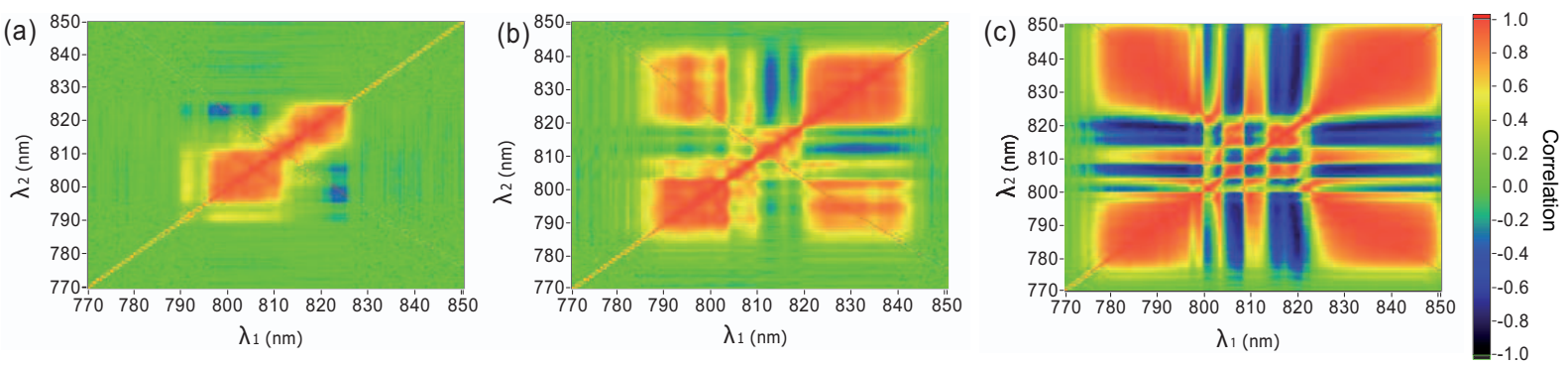

Figure 2. Correlation map within the white-light spectrum at the fixed peak power of $6.5 \mathrm{GW}$

( $\sim$ twice the critical power for filamentation). (a) Before filamentation $(\mathrm{z}=2 \mathrm{~m})$; (b)

Filamentation onset $(\mathrm{z}=3.5 \mathrm{~m})$; (c) Filament end $(\mathrm{z}=8 \mathrm{~m})$. 

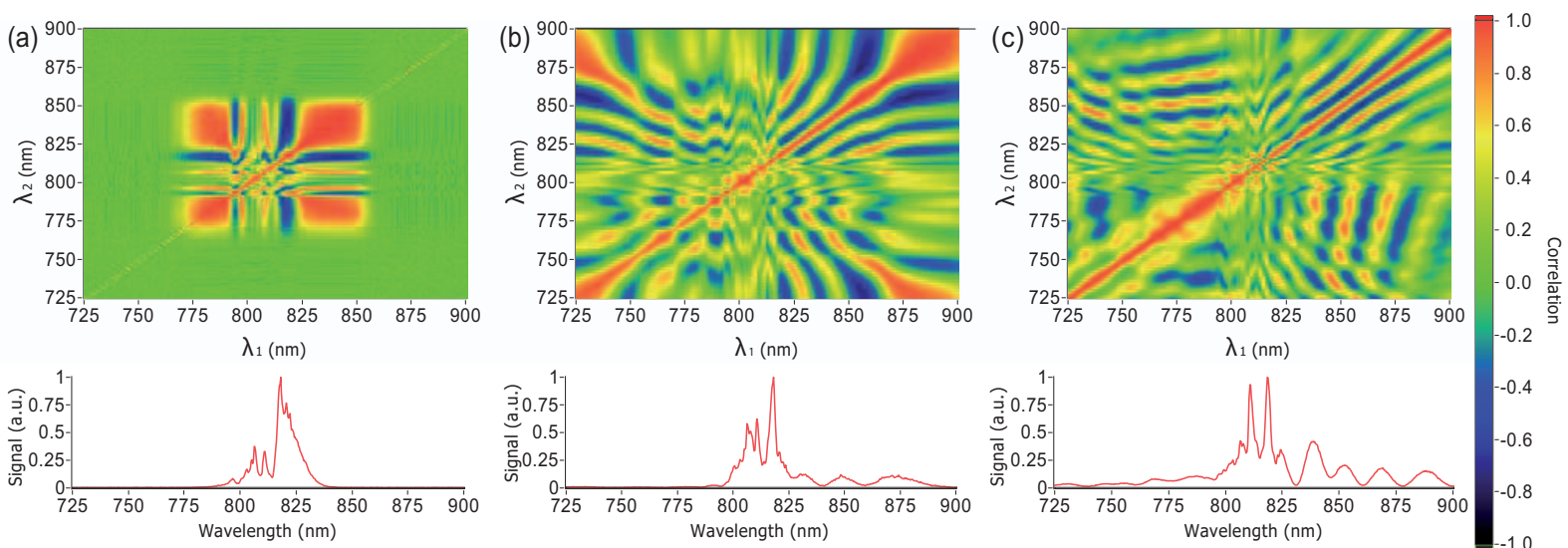

Figure 3. Correlation map within the white-light spectrum at a fixed position $z=12 \mathrm{~m}$, beyond the filament end. (a) $P=6.5 \mathrm{GW}$, twice the critical power for filamentation; (b) $P=9 \mathrm{GW}$; (c) $P=16 \mathrm{GW}$. The panel below each correlation map displays the spectrum of the continuum at the considered power.
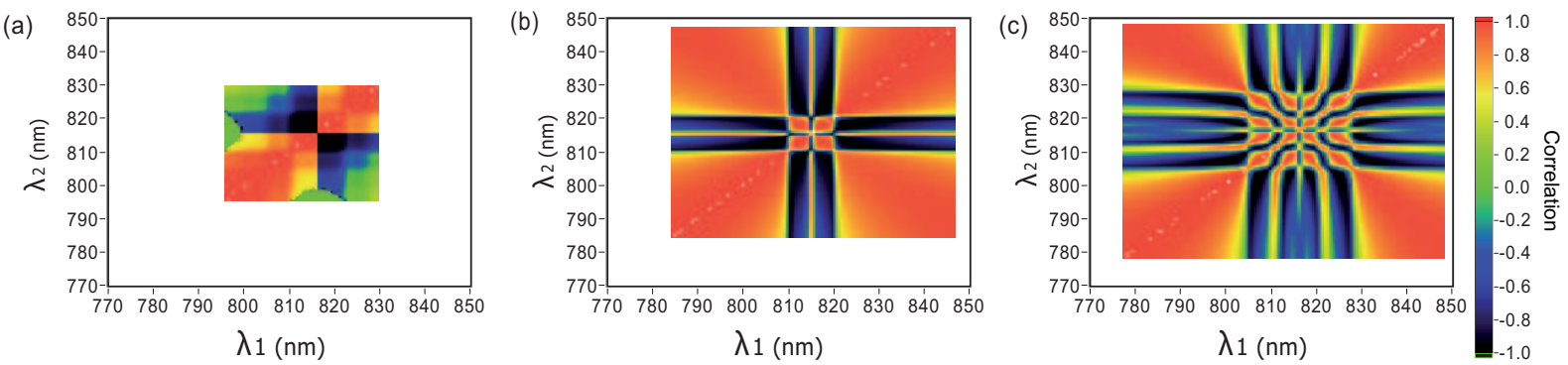

Figure 4. Simulated correlation map. (a) Before filamentation $(z=2 \mathrm{~m})$; (b) Filamentation onset $(z=3.5 \mathrm{~m})$; (c) Filament end $(z=8 \mathrm{~m})$. 